\title{
Architectural Viewpoints for Global Software Development
}

\author{
Bugra M. Yildiz \& Bedir Tekinerdogan \\ Department of Computer Engineering \\ Bilkent University \\ Ankara, Turkey \\ \{bugra,bedir\}@cs.bilkent.edu.tr
}

\begin{abstract}
Global Software Development (GSD) can be considered as the coordinated activity of software development that is not localized and central but geographically distributed. Designing an appropriate software architecture of a GSD system is important to meet the requirements for the communication, coordination and control of the distributed GSD teams. A common practice in software architecture design is to apply architectural views to model the design decisions for the various stakeholder concerns. Unfortunately, existing architectural viewpoint approaches are generalpurpose and not directly dedicated towards GSD projects. In this paper we propose six architectural viewpoints that have been specifically defined to model GSD systems. The architectural viewpoints are based on a meta-model that has been derived after a thorough domain analysis of the GSD literature.
\end{abstract}

Keywords-Global Software Development, Architecture Modeling, Architectural Viewpoint

\section{INTRODUCTION}

Global Software Development (GSD) is a software development approach that can be considered as the coordinated activity of software development that is not localized and central but geographically distributed [8]. The reason behind this globalization of software development stems from clear business goals such as reducing cost of development, solving local IT skills shortage, and supporting outsourcing and offshoring [1]. There is ample reason that these factors will be even stronger in the future, and as such, we will face a further globalization of software development [8].

Designing an appropriate software architecture of a GSD system is important to meet the requirements for the communication, coordination and control of the distributed GSD teams. Software architecture forms one of the key artifacts in the entire software development life cycle since it embodies the earliest design decisions and includes the gross-level components that directly impact the subsequent analysis, design and implementation [5]. A common practice in the software architecture design community is to model and document different architectural views for describing the architecture according to the stakeholders' concerns [5][9][12][13]. An architectural view is a representation of a set of system elements and relations associated with them to support a particular concern. Having multiple views helps to separate the concerns and as such support the modeling, understanding, communication and analysis of the software architecture for different stakeholders. Architectural views conform to viewpoints that represent the conventions for constructing and using a view. An architectural framework organizes and structures the proposed architectural viewpoints. Different architectural frameworks have been proposed in the literature. Examples of architectural frameworks include the Kruchten's 4+1 view model [11], the Siemens Four View Model [9] , and the Views and Beyond approach (V\&B) [5]. In general the existing architectural frameworks tend to be general purpose and not directly focused to a particular domain. The advantage of this is that it can be applied in a broad set of domains, but on the other hand the general-purpose architectural frameworks can fall short for modeling the particular concerns of specific domains.

In this paper we propose an architectural framework including six architectural viewpoints which have been specifically defined for modeling GSD architecture. The architectural framework is based on a meta-model of GSD that we have defined after a thorough domain analysis of the related GSD literature. The meta-model consists of six different parts which each represent the key concepts, i.e. abstract syntax, of the corresponding architectural viewpoint. In addition we have defined the required notation, concrete syntax, that can be used in each architectural viewpoint.

The remainder of the paper is structured as follows. Section II describes the key concerns for architecting GSD and domain specific languages. Section III describes the viewpoints for GSD. Section IV provides a short example illustrating the usage of some GSD viewpoints. Section V describes the related work and finally section VI concludes the paper.

\section{Global SOFTWARE DEVELOPMENT}

Figure 1 shows the conceptual architecture for Global Software Development systems. A GSD architecture usually consists of several nodes, or sites, on which different teams are working to develop a part of the system. The teams could include development teams, testing team, management team etc. Usually each site will also be responsible for following a particular process. In addition, each site might have its own local data storage. 


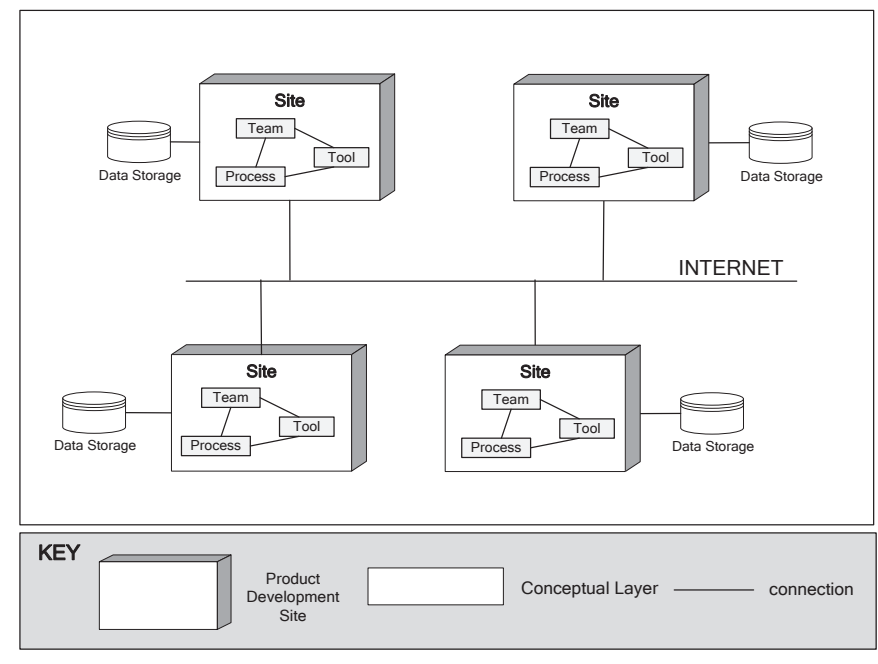

Figure 1. Conceptual Architecture for GSD

Overall the following four important key concerns are identified for designing GSD:

Development - the software development activities typically using a software development process. This includes activities such as requirements analysis, design, implementation and testing. Each site will address typically a subset of these activities.

Communication - the communication mechanisms within and across sites. Typically the different sites need to adopt a common communication protocol to support distributed development.

Coordination - coordination of the activities within and across sites to develop the software according to the requirements. Coordination will be necessary to align the workflows and schedules of the different sites. An important goal could be to optimize the development using appropriate coordination mechanisms.

Control - systematic control mechanisms for analyzing, monitoring and guiding the development activities. This does not only include controlling whether the functional requirements are performed but also which and to what extent quality requirements are addressed.

In fact, each of these concerns requires further in-depth investigation and has also been broadly discussed in the GSD community. The important issue that we would like to address is that each of these concerns can be realized in different ways and likewise will shape the GSD architecture. The GSD software architect needs to identify and define these concerns to design and document the GSD architecture accordingly. To communicate the design decisions, guide the development process and analyze the GSD architecture it is important to model the GSD architecture properly. Unfortunately, each of these concerns are specific to GSD and are not explicitly addressed by existing architectural viewpoint approaches. In the following section we propose the viewpoints for GSD that aim to address the above and other GSD concerns.

\section{Global SOFTWARE DEVELOPMENT VIEWPOINTS}

Defining a new architectural viewpoint implies writing a viewpoint guide. This is similar to the notion of style guide as defined in [5]. The viewpoint guide defines the vocabulary of the architectural element and relation types, and defines the rules for how that vocabulary can be used. For defining a viewpoint guide for a particular quality concern we apply the template as defined in Table 1.

TABLE 1. VIEWPOINT GUIDE TEMPLATE FOR GSD

\begin{tabular}{||l|l||}
\hline Viewpoint Element & \multicolumn{1}{|c||}{ Description } \\
\hline Name & Unique name for the viewpoint \\
\hline Element Types & $\begin{array}{l}\text { The architectural element types } \\
\text { native to the viewpoint }\end{array}$ \\
\hline Relation Types & $\begin{array}{l}\text { The relation types among } \\
\text { architectural elements }\end{array}$ \\
\hline Properties of Elements & $\begin{array}{l}\text { Additional information on the } \\
\text { element types }\end{array}$ \\
\hline Topology Constraints & $\begin{array}{l}\text { The rules of composition of the } \\
\text { elements and relations. }\end{array}$ \\
\hline Notation & $\begin{array}{l}\text { The adopted notation for the } \\
\text { element types and relation types. } \\
\text { The notation can be textual or } \\
\text { visual. }\end{array}$ \\
\hline $\begin{array}{l}\text { Relation to other } \\
\text { views/viewpoints }\end{array}$ & $\begin{array}{l}\text { The relation to other viewpoints } \\
\text { other than the base viewpoint }\end{array}$ \\
\hline \hline
\end{tabular}

A viewpoint defines the template for the views that can be instantiated from it. We consider viewpoints as metamodels representing the basic concepts of the architecture from a particular perspective. To define viewpoints and likewise the viewpoint template, we believe it is necessary to define the corresponding meta-model. Meta-models define the language for the models. In both software language engineering [10] and model-driven development domains [2], a meta-model should have the following two key elements:

Abstract Syntax: Captures the concepts provided by the language and relationships between these concepts.

Concrete Syntax: Defines the notation that facilitates the presentation and construction of models in that language.

Given these key elements of a language, we can also evaluate viewpoints of Global Software Development as the languages for defining GSD views.

Likewise to define the viewpoints for GSD we have to define the corresponding meta-models including both the abstract syntax and the concrete syntax. Based on the literature of GSD, we have defined a meta-model for GSD that defines the concepts and their relations to derive application architecture from different viewpoints. The metamodel consists of six different parts each representing an architectural viewpoint for GSD systems. The viewpoints that we have defined based on the meta-model are Deployment, Process, Data, Communication, Tool and Migration Viewpoints. These viewpoints are described in more detail in the following subsections. For each viewpoint we first provide the abstract syntax, and then the corresponding notation, concrete syntax. 


\section{A. Deployment Viewpoint}

Deployment Viewpoint concerns the deployment of the teams to different sites. The abstract syntax of this viewpoint is shown in Figure 2. Viewpoint guide for Deployment Viewpoint is shown in Table 2.

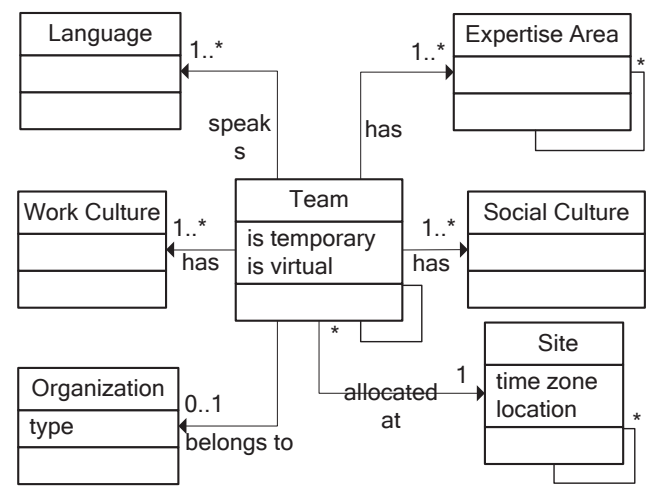

Figure 2. Deployment Viewpoint: Abstract Concrete Syntax

TABLE 2. DEPLOYMENT VIEWPOINT FOR GSD

\begin{tabular}{|c|c|}
\hline "Viewpoint Element & Description \\
\hline Name & Deployment Viewpoint \\
\hline Element Types & $\begin{array}{l}\text { Team, Site, Organization, Language, } \\
\text { Expertise Area, Social Culture, Work } \\
\text { Culture }\end{array}$ \\
\hline Relation Types & $\begin{array}{l}\text { allocated-at, belongs-to, has, speaks, } \\
\text { parent-child }\end{array}$ \\
\hline Properties of Elements & $\begin{array}{l}\text { All elements: name, description, id } \\
\text { Team: isTemporary, isVirtual } \\
\text { Site: timezone, location } \\
\text { Organization: type }\end{array}$ \\
\hline Topology Constraints & $\begin{array}{l}\text { - Team hierarchy must be in the } \\
\text { structure of tree. } \\
\text { - Expertise Area hierarchy must be in } \\
\text { the structure of tree. } \\
\text { - Site hierarchy must be in the } \\
\text { structure of tree. }\end{array}$ \\
\hline \multirow[t]{3}{*}{ Notation } &  \\
\hline & $<$ Properties $>$ \\
\hline & $\begin{array}{l}\text { Language: as property of Team } \\
\text { Expertise Area: as property of Team } \\
\text { Social Culture: as property of Team } \\
\text { Work Culture: as property of Team }\end{array}$ \\
\hline $\begin{array}{l}\text { Relation to other } \\
\text { views/viewpoints }\end{array}$ & $\begin{array}{l}\text { - Team entity is common for all } \\
\text { viewpoints. } \\
\text { - Expertise Area entity is used in } \\
\text { Process viewpoint. }\end{array}$ \\
\hline
\end{tabular}

Team is the primary essential entity in the viewpoint and is defined as a group of persons that work together to achieve a particular goal. Team may be organized in a temporary way and be dismissed after its function is complete. Team is allocated at a particular Site. Site may be located in a country, city or a building where a Team works at. The attribute location determines where Site is placed in the world. Time zone shows the local time of Site. Teams may belong to different types of Organizations, such as commercial organizations, subcontractors or non-profitable organizations such as open source communities. Teams can be from different countries and depending on the society they are in, they may have different Social Cultures. Team may further include Work Culture including work experience, the time that members work together, their habits etc. Expertise Area, Team and Site can be further decomposed into sub-parts. For example, a Software Team may consist of sub-Teams each responsible for Design, Implementation, Testing and Integration.

\section{B. Process Viewpoint}

Process Viewpoint concerns the different kind of processes in GSD. The abstract syntax of Process unit is shown in Fig 3. Viewpoint guide for this viewpoint is shown in Table 3.

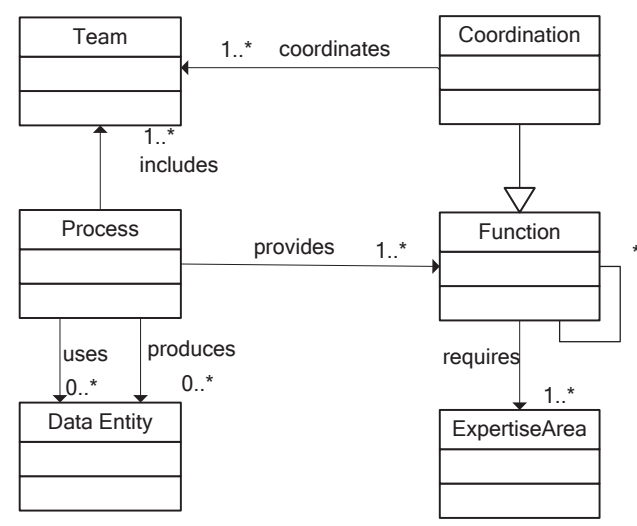

Figure 3. Process Viewpoint: Abstract Syntax

TABLE 3. PROCESS VIEWPOINT FOR GSD

\begin{tabular}{|c|c|}
\hline Viewpoint Element & Description \\
\hline Name & Process Viewpoint \\
\hline Element Types & $\begin{array}{l}\text { Team, Coordination, Process, Function, } \\
\text { Data Entity, Expertise Area }\end{array}$ \\
\hline Relation Types & $\begin{array}{l}\text { coordinates, includes, provides, uses, } \\
\text { produces, requires, parent-child, extends }\end{array}$ \\
\hline Properties of Elements & All elements: name, description, id \\
\hline Topology Constraints & $\begin{array}{l}\text { - Function hierarchy must be in the } \\
\text { structure of tree. }\end{array}$ \\
\hline \multirow[t]{5}{*}{ Notation } & 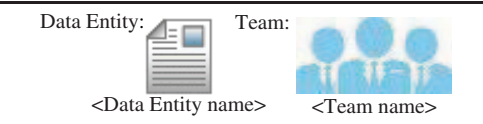 \\
\hline & Function: \\
\hline & $f<$ Function name $>\quad \xi_{\text {S }}<$ Process name $>$ \\
\hline & $<$ Expertise Areas $>$ \\
\hline & $\begin{array}{c}\text { Function-Process association: } \\
<\text { Function }>\rightarrow<\text { Process }> \\
\text { Data Entity-Process association: } \\
\quad<\text { Used Data Entity }>\rightarrow<\text { Process }> \\
<\text { Process }>\longrightarrow<\text { Produced Data Entity }> \\
\text { Expertise Area: as property of Function }\end{array}$ \\
\hline $\begin{array}{l}\text { Relation to other } \\
\text { views/viewpoints }\end{array}$ & $\begin{array}{l}\text { - Team entity is common for all } \\
\text { viewpoints. } \\
\text { - Expertise Area entity is used in } \\
\text { Deployment viewpoint. } \\
\text { - Data Entity entity is used in Data } \\
\text { viewpoint. }\end{array}$ \\
\hline
\end{tabular}


Process is defined as a planned set of activities that aims to provide some service. Teams participate in Process in order to provide some service. Service is defined with Function. A Function can be any service during software development process that requires some Expertise Areas such as software development, architecture design, business management, requirements elicitation and so on. Coordination is also a Function that should be provided for coordinating several Teams' activities. A Process consumes or uses several different Data Entities and also creates other Data Entities for providing targeted Functions. For supporting activities defined in Process, Process concept is further specialized into Workflow, Business Process and Development Process (not shown in figure).

\section{Data Viewpoint}

Data Viewpoint is for representing ownership and physical deployment of software development data. The abstract syntax is shown in Figure 4. Table 3 shows the viewpoint guide for Data Viewpoint.

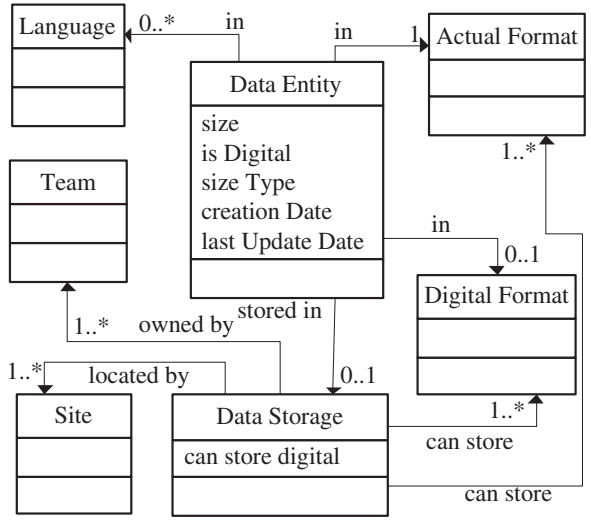

Figure 4. Data Viewpoint: Abstract Syntax

Data Entity is the fundamental entity of this viewpoint. It represents any piece of data: digital, textual or informal piece of information such as notes taken by developers, telephone calls that are usually not recorded. Data Entity has size whose unit is defined by size type; for example, a 120-page report, 6 minutes of voice record, 2 gigabyte of digital data. Creation date and last update date show the history of Data Entity. Data Entity has Actual Type where Actual Format can be one of predefined formats (video, sound, text, picture and complex-Data Entity) or some designer defined format. If Data Entity is digital, then in addition to Actual Format, it has a Digital Format. Data Entity may be implemented in one or more Languages.

Data Entity is stored in Data Storage. Data Storage corresponds to any object in real world that can store information. For example, some textual document is stored in paper form, or it is stored in a voice record, or it is stored digitally in the format of some text editor. Data Storage has ability to store some Actual Types and if it can store digital data, then it can support some Digital Types also. A Data Storage instance is owned by one or more Teams and it can be located in one Site or may be distributed over several Sites like distributed databases.

TABLE 4. DATA VIEWPOINT FOR GSD

\begin{tabular}{|c|c|}
\hline Viewpoint Element & Description \\
\hline Name & Data Viewpoint \\
\hline Element Types & $\begin{array}{l}\text { Language, Team, Site, Data Storage, } \\
\text { Digital Format, Actual Format, Data } \\
\text { Entity }\end{array}$ \\
\hline Relation Types & $\begin{array}{l}\text { in, owned by, stored in, located by, can } \\
\text { store }\end{array}$ \\
\hline Properties of Elements & $\begin{array}{l}\text { All elements: name, description, id } \\
\text { Data Storage: canStoreDigital } \\
\text { Data Entity: size, isDigital, sizeType, } \\
\text { creationDate, lastUpdateDate }\end{array}$ \\
\hline Topology Constraints & $\begin{array}{l}\text { - Data Storage can store Digital Format } \\
\text { only if Data Storage can store digital. } \\
\text { - Data Entity is in some Digital Format } \\
\text { only if Data Entity is digital. }\end{array}$ \\
\hline \multirow[t]{8}{*}{ Notation } & $\begin{array}{l}\text { Site: } \\
\begin{array}{|c|}<\text { CSite name }> \\
<\text { Data Storages }>\end{array}\end{array}$ \\
\hline & $<$ Team name $>$ \\
\hline & 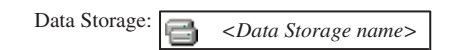 \\
\hline & $<$ Compatible Formats $>$ \\
\hline & $<$ Data Entities $>$ \\
\hline & Data Entity: 望 <Data Entity name $>$ \\
\hline & $<$ Compatible Formats $>$ \\
\hline & $\begin{array}{l}\text { Actual-Digital Format: } 0<\text { Format name }> \\
\text { Team-Data Storage association: } \\
<\text { Data Storage }>\longrightarrow<\text { Team }>\end{array}$ \\
\hline $\begin{array}{l}\text { Relation to other } \\
\text { views/viewpoints }\end{array}$ & $\begin{array}{l}\text { - Team entity is common for all } \\
\text { viewpoints. } \\
\text { - Data Entity entity is used Process and } \\
\text { Communication viewpoints. } \\
\text { - Data Storage is used in Migration } \\
\text { viewpoint. } \\
\text { - Actual and Digital Formats are used } \\
\text { in Tool Viewpoint. }\end{array}$ \\
\hline
\end{tabular}

\section{Communication Viewpoint}

Communication Viewpoint focuses on the representation of both formal and informal communication activities between Teams. The abstract and concrete syntax are shown in Figure 5. Table 5 displays the viewpoint guide.

Communication is done over Communication Platform in the context of Process and it can be an instance of sudden/event based communication activity like a telephone call or a continuous communication channel such as a discussion forum. Type attribute is for representing in which way Communication takes place such as email, phone call, face-to-face chat and so on. Suggested time period is an important attribute for GSD since Teams work in different time zones, some Communication channels can be used effectively in a defined time period. For example, phone calls should be done during the hours when both sides are in or around their work hours. 


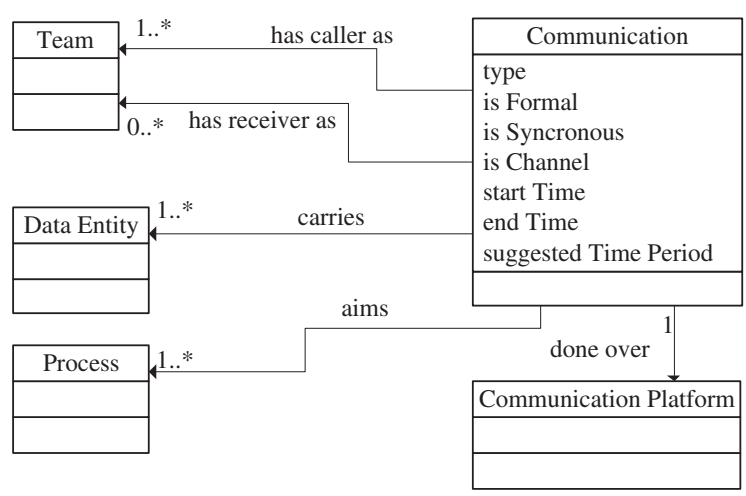

Figure 5. Communication Viewpoint: Abstract Syntax

TABLE 5. COMMUNICATION VIEWPOINT FOR GSD

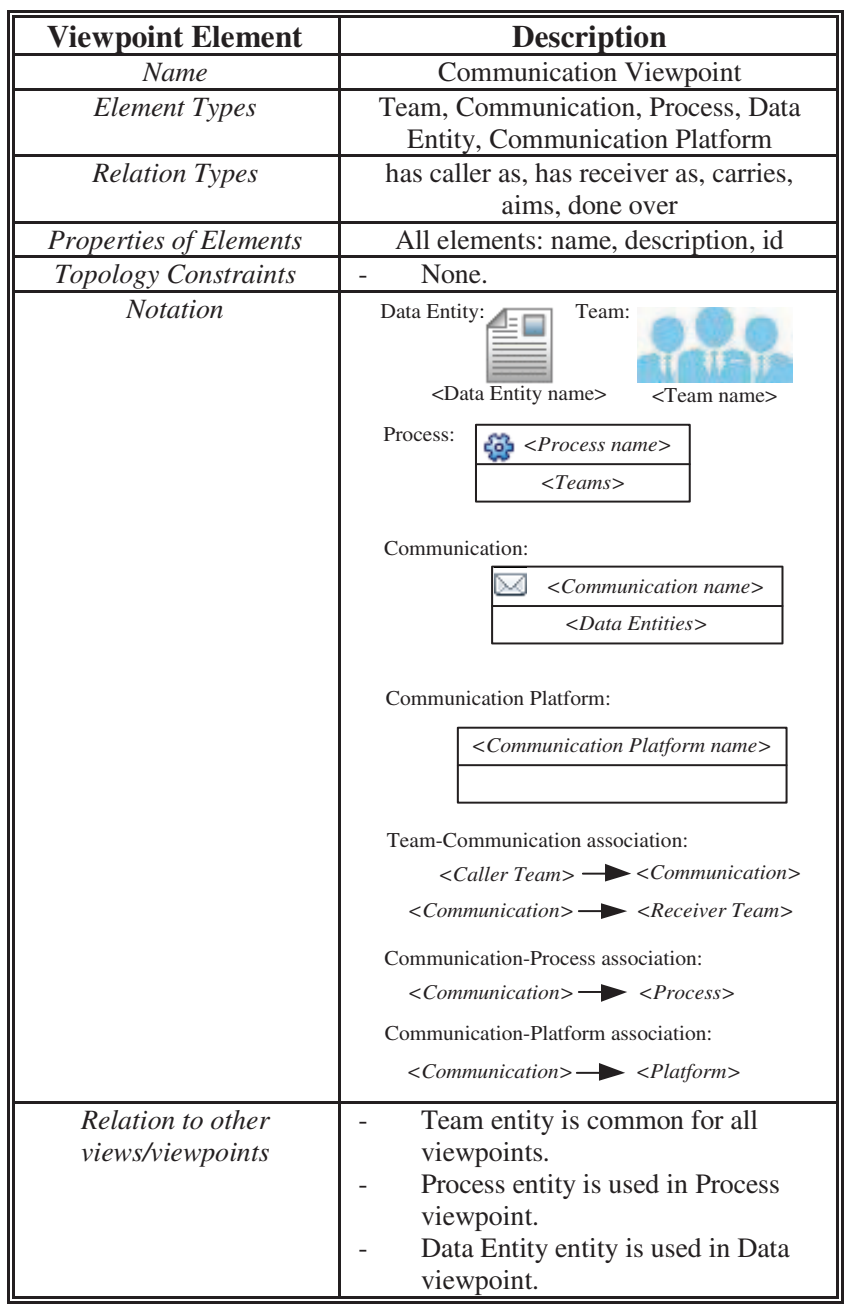

Communication has two sides which are caller and receiver. Generally speaking, caller starts communication and receiver is the one who is called by caller. For example, an email sender is classified as caller and receiver is the one who receives email. Sometimes, there can be multiple callers such as video conferences or there can be multiple receivers such as discussion forums. It is also possible that caller and receiver are the same such as a planned meeting. For all cases, caller and receivers are considered as Teams in this viewpoint. While Teams communicate, one or more Data Entities are carried in the context of Communication.

\section{E. Tool Viewpoint}

Tool Viewpoint captures details of tools used by Teams for communication and providing Functions. The abstract syntax is shown in Figure 6 and viewpoint guide is shown .n Table 5.

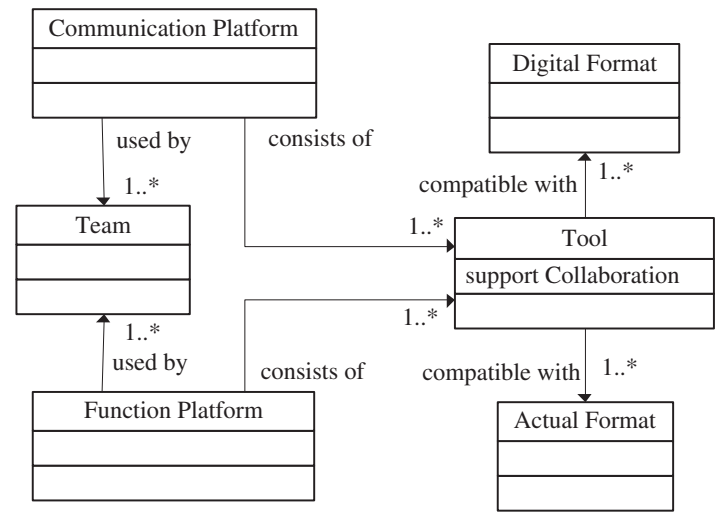

Figure 6. Tool Viewpoint: Abstract Syntax

Tool is compatible with one or more Actual Format and Digital Format. Platform is the set of Tools used by Teams for communication or providing some functions. Depending on the purpose, the platform is defined as Function Platform or Communication Platform.

TABLE 6. TOOL VIEWPOINT FOR GSD

\begin{tabular}{|c|c|}
\hline Viewpoint Element & Description \\
\hline Name & Tool Viewpoint \\
\hline Element Types & $\begin{array}{c}\text { Communication Platform, Function } \\
\text { Platform, Team, Digital Format, Actual } \\
\text { Format, Tool }\end{array}$ \\
\hline Relation Types & used by, consists of, compatible with \\
\hline Properties of Elements & $\begin{array}{l}\text { All elements: name, description, id } \\
\text { Tool: supportCollaboration }\end{array}$ \\
\hline Topology Constraints & None. \\
\hline \multirow[t]{5}{*}{ Notation } & $\begin{array}{l}\text { Tool: } \\
\begin{array}{|l|}<\quad<\text { Tool name }> \\
<\text { Compatible Formats }> \\
\end{array}\end{array}$ \\
\hline & $<<\mathrm{Te}$ \\
\hline & $<$ Platform name $>$ \\
\hline & $<$ Tools $>$ \\
\hline & $\begin{array}{l}\text { Actual-Digital Format: }<\text { Format name }> \\
\text { Team-Platform association: } \\
<\text { Platform }>\longrightarrow<\text { Team }>\end{array}$ \\
\hline $\begin{array}{l}\text { Relation to other } \\
\text { views/viewpoints }\end{array}$ & $\begin{array}{ll}\text { - } & \text { Team entity is common for all } \\
\text { viewpoints. } \\
\text { - Actual and Digital Formats are used } \\
\text { in Data Viewpoint. } \\
\text { Communication Platform is used in } \\
\text { Communication Viewpoint. }\end{array}$ \\
\hline
\end{tabular}




\section{F. Migration Viewpoint}

Migration Viewpoint concerns the migration and traveling of Teams during GSD activities. These travels are especially needed in the first and final phases of the projects to ease and support coordination and integration. Figure 7 shows the abstract syntax of Migration Viewpoint. Viewpoint guide for Migration Viewpoint is shown in Table 6.

Migration is executed by one or more Teams from Site to Site at a particular date. In a Migration, Teams may carry Data Storage such as documents, digital data containers and so on. Migration is executed in the context of Process.

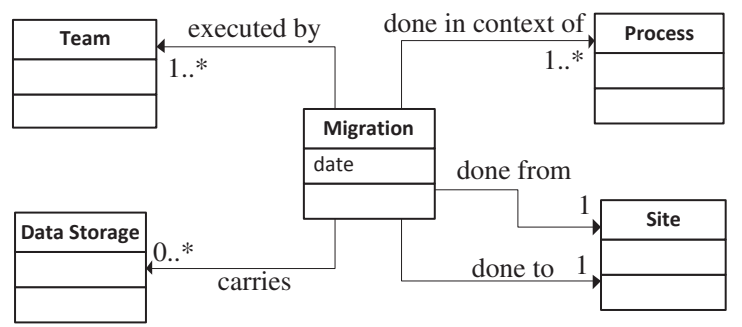

Figure 7. Migration Viewpoint: Abstract Syntax

TABLE 7. MigRATION VIEWPOINT FOR GSD

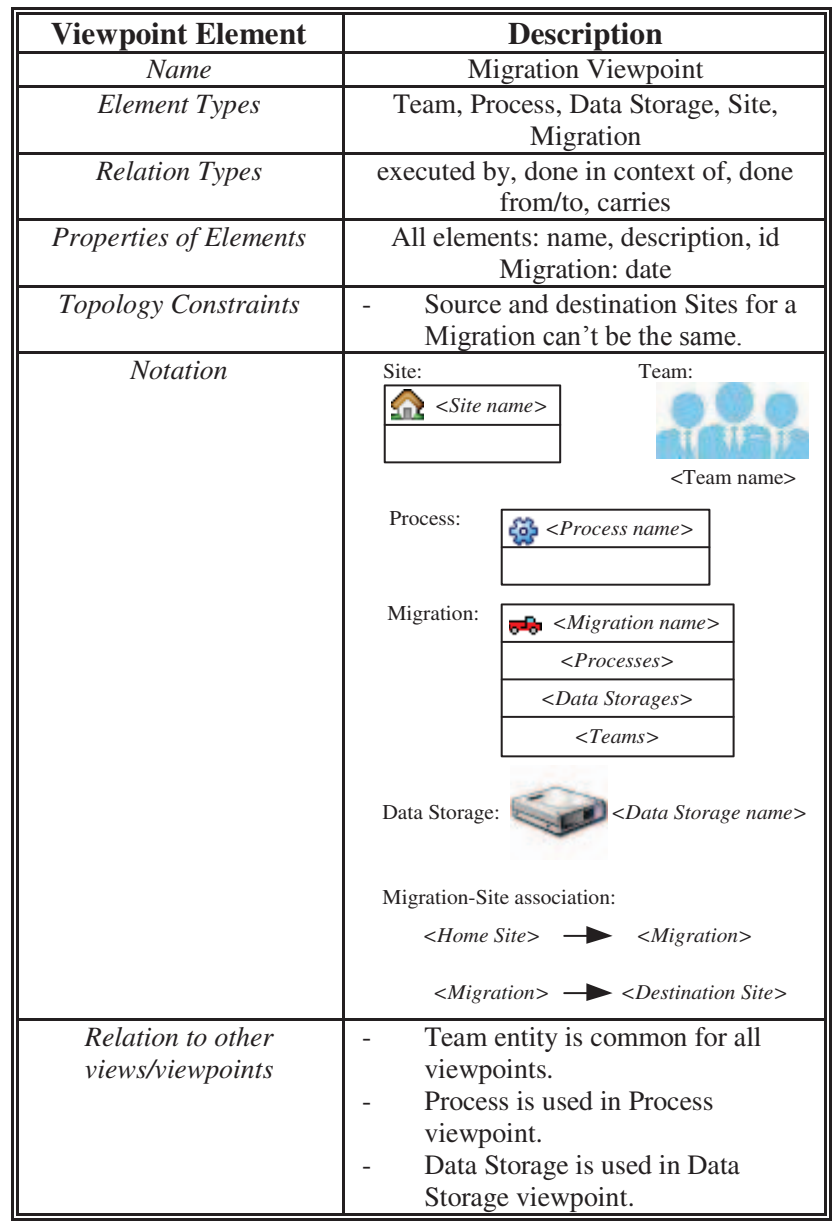

\section{EXAMPLE CASE}

As an example case, consider a GSD project with 7 Sites located at different places in the world. Company A, the owner of the project, has its center operating in United States. Requirement Analysis is done in New York and Architecture Team works in Silicon Valley, California. Company A has development center in New Delhi, India where software development and test Teams work. Also, Company A works with a subcontractor company, Company B for some particular components. Company B's center is located in Sao Paulo, Brazil. Company B also has development and test Teams. Figure 8 shows the Deployment View of this case description. The Deployment View is instantiated from the Deployment Viewpoint in Table 2.

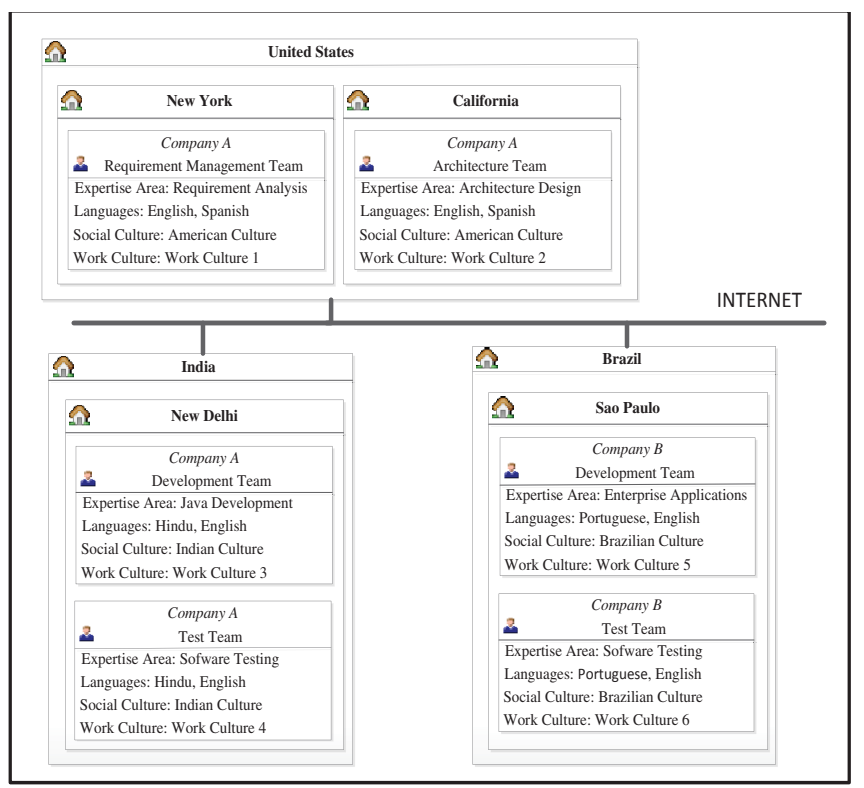

Figure 8. Example Case: Deployment View

For the example case described in the previous paragraph, we also give an example process flow. Requirement Management Team follows a Requirement Analysis process which is done for serving Requirement Derivation function. The output of Requirement Analysis process is Requirement Description Document. This document is used by Architecture Team in order to design architecture. The document is an input to Architecture Design process and this process creates Architecture Description Document which then be used in implementation process. Since there are two Sites taking role in development, Architecture Description Document is an input to two different development processes whose outputs are working software units. This process flow is shown in Process View in Figure 9. The Process View is instantiated from the Process Viewpoint definition of Table 3. We have not included some other details of this process flow such as the causal connections among the different steps. 


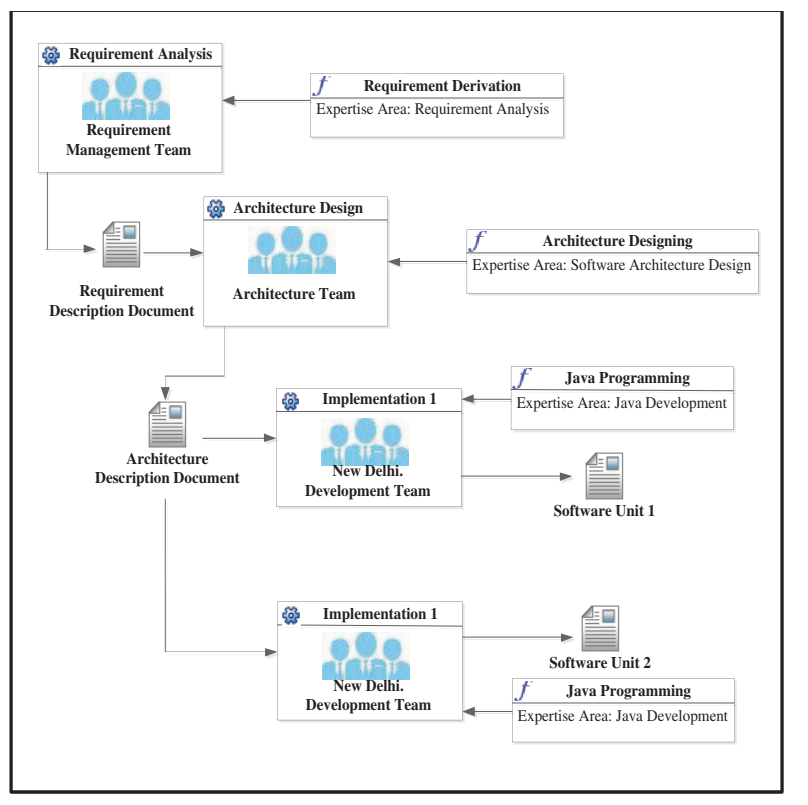

Figure 9. Example Case: Process View

The Migration view for the given case is given in Figure 10. As we stated before, migrations from site to site take place especially in the phases of the projects which require extensive collaboration and coordination. The Migration view illustrates the case in which a team in New York migrates to California. The travel is done in the context of Requirement Management and Architecture Design processes. Requirement Team also carries an external disk in order to carry some information that may be needed such as requirements documentations, some work templates, etc.

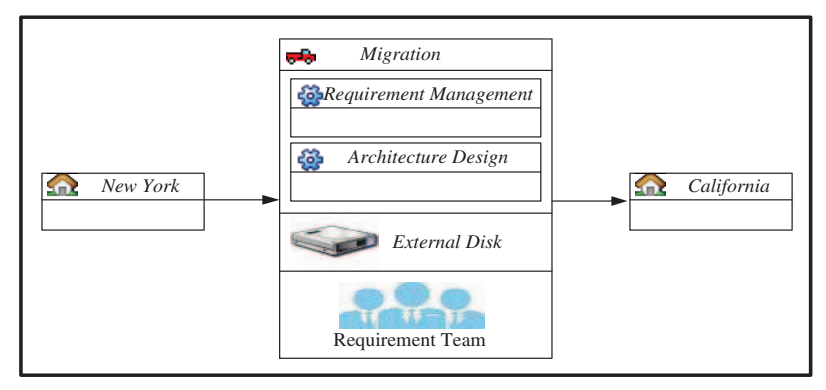

Figure 10. Example Case: Migration View

Figure 11 shows the Data view for the given example. The view shows the three sites New York, California, New Delhi and Sao Paulo which have their own local data storages. Further, each site has its own documents and local copies of other necessary documents kept in these local storages.

Due to lack of space we have only given four views. The designer can also use the other four viewpoints to define the corresponding views.

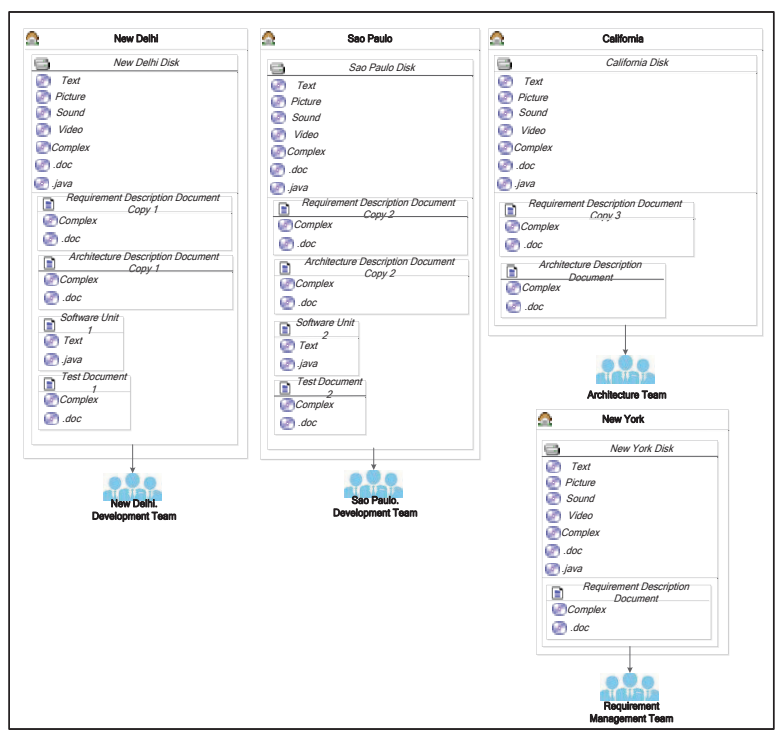

Figure 11. Example Case: Data View

\section{RELATED WORK}

Notably, architecting in GSD has not been widely addressed. The key research focus in the GSE community seems to have been in particular related to tackling the problems related to communication, coordination and control concerns.

Clerk et al. [6] report on the use of so-called architectural rules to tackle the GSD concerns. Architectural rules are defined as "principles and statements about the software architecture that must be complied with throughout the organization". They have defined four challenges in GSD: time difference and geographical distance, culture, team communication and collaboration, and work distribution. For each of these challenges they list possible solutions and describe to what extent these solutions can be expressed as architectural rules. The work of Clerk et al. aims to shed light on what kind of architectural rules are necessary to guide the GSD. We consider our work complementary to this work. In our work the design actions that relate to the expected answers of questions are defined as design actions.

Avritzer et al. [2] report on their experience in assessing the relationship between the dependency structure of a software architecture and the coordination needs among distributed development teams. They have used matrix models in the Global Studio Project Version 3.0, to represent both architectural dependencies and the coordination structure among the team members. The analysis of data gathered during the Global Studio Project Version 3.0 showed that design structure matrix (DSM) models representing the software architecture of GSD can help guide the task assignments in global software development projects.

Tool support has been named as one of the important challenges for GSD since it requires making software development tools and environments more collaborative [14]. Booch and Brown have introduced the vision for Collaborative Development Environment (CDE), which is 
defined as "a virtual space wherein all the stakeholders of the project - even if distributed by time or distance - may negotiate, brainstorm, discuss, share knowledge, and generally labor together to carry out some task, most often to create an executable deliverable and its supporting artifacts [3]. A number of efforts have been carried out to support the idea of CDEs. Collab.net [7] is a commercial provider of CDEs, offering facilities for configuration management, bug tracking, task management and discussions. Spanjers et al. [15] discuss the system SoftFab, which automates the build and test processes in the context of multi-site projects. Caroll et al. [4] define the tool Jazz which supports rich synchronous communication, and promotes mutual awareness of coding activities within a development team. We believe that a common metamodel for GSD could help developing CDEs. The metamodel that we have provided in this paper could serve this purpose.

To support coordination among sites, usually it is aimed to adopt the same development and execution platform. Unfortunately, adopting a single platform might not be always possible due to technical or organizational constraints of the different sites in GSD projects. As such, very often GSD projects have to cope with portability and interoperability problems. We have addressed these issues in our earlier work [16]. Here, we have proposed to apply a model-driven architecture design (MDA) approach to abstract away from platform concerns. Based on model transformation patterns as defined by MDA the portability and the interoperability concerns can be more easily addressed. The viewpoints in this paper could be uses as the source and target meta-models in the model transformation patterns.

\section{CONCLUSION}

Different challenges have been identified to set up a Global Software Development environment. Our literature study on GSD showed that in particular the challenges of communication, coordination, and control of GSD is addressed in the GSD community but less focus has been provided on the modeling, documentation and analysis of architecture for GSD. In this paper we have focused on the architecture design of GSD. Designing architecture for single systems is hard. Designing architecture for GSD is even more difficult due to the additional concerns for communication, coordination and control of distributed GSD teams. To support the architect in designing a proper GSD architecture we have provided a meta-model for GSD based on a thorough literature study. The meta-model captures the key concerns for designing GSD software architecture. We have identified the six different concerns including deployment, process, data, communication, tool and control. The meta-model served as a foundation for defining the architectural viewpoints for GSD. In our future work we plan to apply the approach in a real industrial setting. Also we aim to provide tool support dedicated towards modeling GSD architectures.

\section{REFERENCES}

[1] Agerfalk, P.J., B. Fitzgerald, H. Holmström Olsson, and E.O' Conchuir. Benefits of Global Software Development: The Known and Unknown. in International Conference on Software Process, pp. 1-9, 2008.

[2] A. Avritzer, D. Paulish, Y. Cai. Coordination Implications of Software Architecture in a Global Software Development Project, Seventh Working IEEE/IFIP Conference on Software Architecture, pp. 107-116, 2008.

[3] G. Booch and A. Brown. Collaborative Development Environments. Advances in Computers Vol. 59, Academic Press, August, 2003.

[4] M. Carroll and S. Sprenkle. Coven: Brewing Better Collaboration through Software Configuration Management. ACM SIGSOFT Foundations of Software Engineering, pp. 88-97, 2000.

[5] P. Clements, F. Bachmann, L. Bass, D. Garlan, J. Ivers, R. Little, P. Merson, R. Nord, J. Stafford. Documenting Software Architectures: Views and Beyond. Second Edition. AddisonWesley, 2010.

[6] V. Clerc, P. Lago, H. van Vliet. Global Software Development: Are Architectural Rules the Answer? In: Proc. of the 2nd International Conference on Global Software Engineering, pp. 225-234. IEEE Computer Society Press, Los Alamitos, 2007.

[7] Collab.net http://www.collab.net, http://sourceforge.net, accessed June, 2011

[8] J.D. Herbsleb, Global Software Engineering: The Future of Socio-technical Coordination, 2007 Future of Software Engineering, p.188-198, May 23-25, 2007.

[9] C. Hofmeister, R. Nord, and D. Soni. Applied Software Architecture. Addison-Wesley, NJ, USA.

[10] A. Kleppe. Software Language Engineering: Creating Domain-Specific Languages Using Meta-models. AddisonWesley Longman Publishing Co., Inc., Boston, 2009.

[11] P. Kruchten. The 4+1 View Model of Architecture. IEEE Software, 12(6):42-50, 1995.

[12] A.J. Lattanze. Architecting Software Intensive Systems: A Practitioner's Guide, Auerbach Publications, 2009.

[13] R. Nord, P. Clements, D. Emery, R. Hilliard. A Structured Approach for Reviewing Architecture Documentation, Technical Note, CMU/SEI-2009-TN-0302009, SEI-CMU, 2009.

[14] B. Sengupta, S. Chandra, V. Sinha. A research agenda for distributed software development, In Proceedings of the 28th international conference on Software engineering, pp. 731740, 2006.

[15] H. Spanjers, Ter, B. Graaf, M. Lormans, D. Bendas, R. V. Solingen. Tool Support for Distributed Software Engineering, in Proc of: International Conference on Global Software Engineering, 2006. ICGSE '06., pp. 187-198, 2006.

[16] B. Yildiz \& B. Tekinerdogan. Meta-Model For Global Software Development to Support Portability and Interoperability, Sixth International Conference on Software Engineering Advances (ICSEA 2011), Barcelona, Spain, October, 2011. 